\title{
Evidence for Active Atomic Defects in Monolayer Hexagonal Boron Nitride
}

\author{
Ovidiu Cretu ${ }^{1}$, Yung-Chang Lin $^{1}$ and Kazutomo Suenaga ${ }^{1}$ \\ ${ }^{1 .}$ National Institute of Advanced Industrial Science and Technology (AIST), Nanotube Research Center, \\ Central 5, 1-1-1 Higashi, Tsukuba, Ibaraki 305-8565, Japan.
}

Mechanical behavior is an important issue in low-dimensional materials both from a fundamental physics point of view as well as in view of their future applications. There has been little work done on atomic-scale defects in h-BN; in particular, no experimental evidence has been so far reported for activated defect structures in h-BN, which could be responsible for the plastic deformation of the h-BN layer. The lack of evidence for plasticity is mainly due to the difference in bond nature between h-BN and graphene. The ionic character of the B-N bond will not allow bond switching as easily as in the case of C-C bonds. Here we show the first experimental evidence for activated atomic defects in single-layer h-BN at elevated temperatures under e-beam irradiation. The $4 \mid 8$ defects are stabilized with homoelemental B-B and N-N bonds and are mobile in the h-BN lattice. Unlike the case of a graphene layer, in which the Stone-Wales transformation is responsible for the plastic deformation, the deformation of $\mathrm{h}-\mathrm{BN}$ seems to rely on atomic row gliding along the armchair direction. This represents a new mechanism of plasticity for this material [1].

The $4 \mid 8$ defects were observed along the armchair direction of single-layered regions of $\mathrm{h}-\mathrm{BN}$ during TEM observation at $700^{\circ} \mathrm{C}$ (Fig. 1a). One can see alternating smaller and larger rings, corresponding to the four and eight membered polygons respectively. There are two possibilities for the atomic arrangement of boron and nitrogen in the $4 \mid 8$ defects, namely "symmetric" (Fig. 1b) and "asymmetric" (Fig. 1c). In the asymmetric case, all the squares and octagons contain exclusively B-N bonds. The $4 \mid 8$ structure found in our experiments can only be explained by the symmetric configuration, involving B-B and N-N homoelemental bonding. The asymmetric structure can only exist between two different h-BN domains with a $60^{\circ}$ rotation between them and thus cannot form in a single h-BN domain.

Experimental evidence for the motion of these structures is presented in Figure 2, which shows groups of TEM images extracted from image series acquired of the $4 \mid 8$ defects. Panel (a) shows a process in which an initially straight defect undergoes a transformation. This results in a structure which shows a small vertical step between its two parts, but is otherwise still continuous. A second step is seen to appear in the right-hand side frame in (a), corresponding to an additional transformation. Panel (b) shows a $4 \mid 8$ defect that undergoes a two-step process. From left to right, the initially continuous $4 \mid 8$ defect separates into sections which are offset in a direction perpendicular to the defect axis. The two parts become further separated following a second transformation, which increases the distance between them both horizontally and vertically.

The diffusion of the $4 \mid 8$ defects can be explained through the glide of one or two atomic rows along the armchair direction. A schematic of this process is given in Figure 3, where the three panels highlight the different types of motion that we observe. Because of the way in which two of these translations bring the two resulting $4 \mid 8$ defects closer together or farther apart, they are called "inward" (a) and "outward" (b), respectively. The third one is called "perpendicular" (c), because of its orientation with respect to the $4 \mid 8$ defect axis. Macroscopically, these motions are equivalent to gliding part of the h-BN lattice along the three non-equivalent zig-zag directions. The h-BN lattice accommodates these glides through 
the formation of pentagon and heptagon rings.

Our findings represent the first observation of plasticity in this material and open the way for further studies involving it, as well as other 2D analogues [2].

\section{References:}

[1] O Cretu, Y-C Lin and K Suenaga, Nano Lett. DOI: 10.1021/nl404735w (2014).

[2] This work was supported by a JST Research Acceleration program.
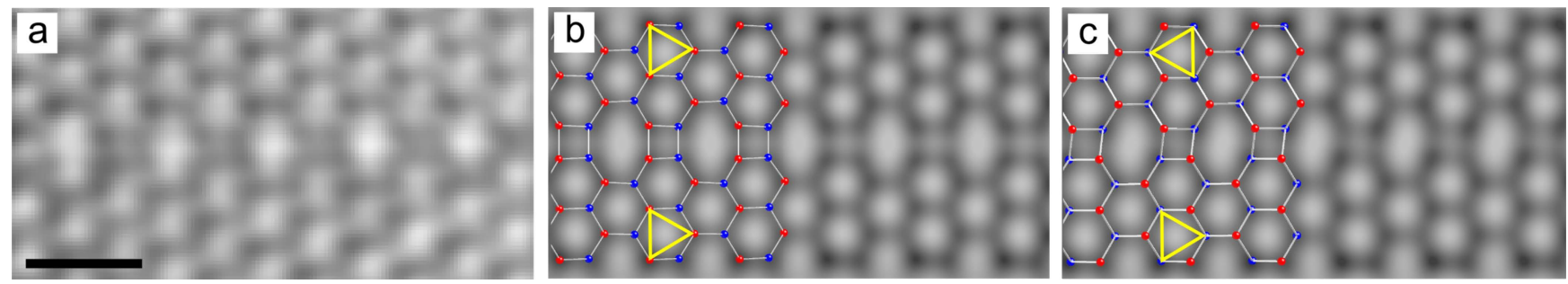

Figure 1. (a) HR-TEM image of a $4 \mid 8$ line defect. (b) Simulated image and corresponding atomic model of the "symmetric" case. (c) Simulated image and corresponding atomic model of the "asymmetric" case. Boron and nitrogen atoms are colored red and blue, respectively. Scale bar is $0.5 \mathrm{~nm}$.

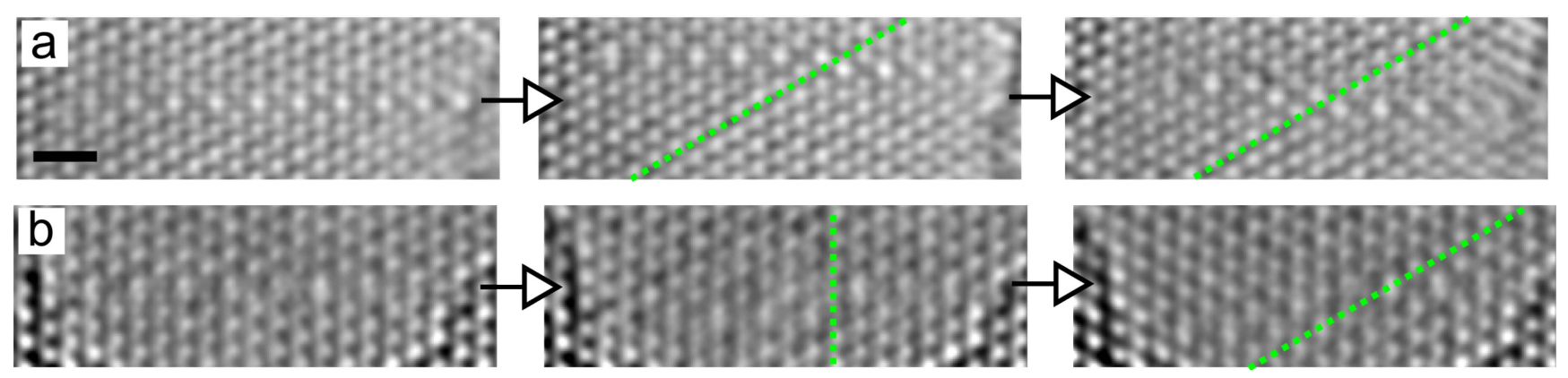

Figure 2. Experimental evidence of $4 \mid 8$ defect motion. Panels (a) and (b) show series of frames highlighting trajectories for different $4 \mid 8$ defects. Scale bar is $0.5 \mathrm{~nm}$.
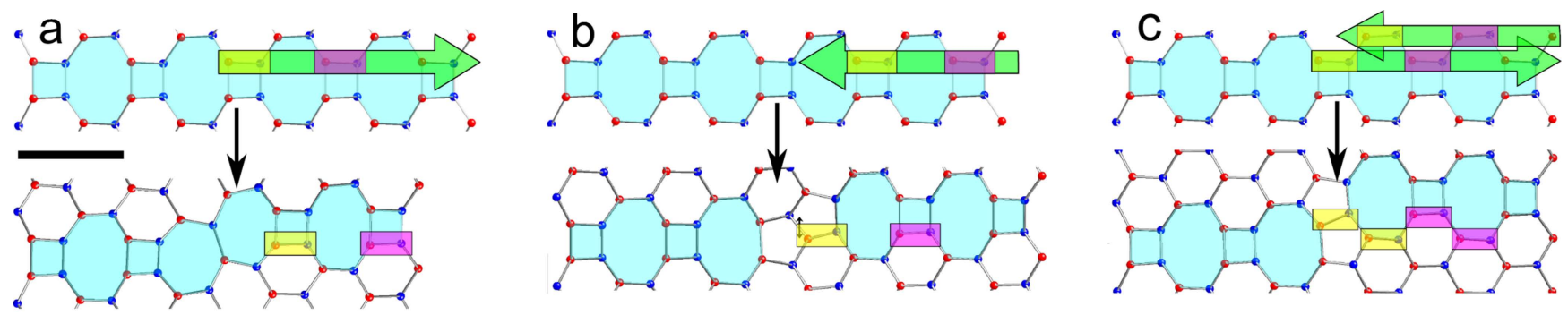

Figure 3. $4 \mid 8$ defect motion through atomic-row glides. Panels (a)-(c) show the initial and optimized structure following a movement in each of the cases. The atoms which will move are indicated by a green arrow. Boron and nitrogen atoms are colored red and blue, respectively. Scale bar is $0.5 \mathrm{~nm}$. 